\title{
Avaliação dos resultados clínicos após cirurgia descompressiva em cães com doença de disco intervertebral
}

[Evaluation of clinical results of decompressive surgery in dogs with degenerative disk disease]

\author{
M.V.B. Arias $^{1}$, C.M. Nishioka ${ }^{2}$, C.O. Garcia ${ }^{2}$, A.Z. Reia ${ }^{3}$, D. Baraúna Júnior ${ }^{2}$, R.A. Marcasso ${ }^{4}$ \\ ${ }^{1}$ Universidade Estadual de Londrina \\ Caixa Postal 6001 \\ 86051-990 - Londrina \\ ${ }^{2}$ Médico veterinário autônomo - \\ ${ }^{3}$ Médica veterinária - Hospital Veterinário - UEL \\ ${ }^{4}$ Médico veterinário - Residente - UEL
}

\begin{abstract}
RESUMO
Avaliaram-se os resultados clínicos após realização de cirurgia descompressiva em 45 cães com doença do disco intervertebral cervical ou toracolombar. Após a cirurgia, 35 cães $(77,8 \%)$ recuperaram-se totalmente, oito $(17,8 \%)$ parcialmente e dois $(4,4 \%)$ não apresentaram alteração do quadro inicial. Em oito cães com paraplegia e perda da sensibilidade dolorosa profunda houve completa melhora do quadro clínico, com recuperação total em $62,5 \%$ dos casos. Em quatro cães com tetraparesia, a cirurgia foi eficaz. A cirurgia descompressiva (slot cervical e hemilaminectomia toracolombar), com a retirada do material do disco do interior do canal vertebral, foi uma forma efetiva de gerar melhora do quadro funcional.
\end{abstract}

Palavras-chave: cão, sistema nervoso, doença do disco intervertebral

\begin{abstract}
Clinical results after decompressive surgery were evaluated in 45 dogs with cervical or thoracolumbar intervertebral disk disease. After surgery, 35 dogs recovered totally, eight (17.8\%) partially, and two (4.4\%) did not present any change in clinical findings. Eight dogs with paraplegy and loss of deep pain perception showed improvement, with total recovering in $62.5 \%$ of cases. Surgery was effective in four dogs with tetraparesy. Decompressive surgery (cervical slot or hemilaminectomy), with removal of disk material from inside the vertebral canal, was an effective form to produce functional improvement in dogs with this disease.
\end{abstract}

Keywords: dog, nervous system, intervertebral disk disease

\section{INTRODUÇÃO}

A doença do disco intervertebral (DDIV) é uma das causas mais comuns de alterações neurológicas em cães (Amsellem et al., 2003a). É uma afeç̧ão provocada pela degeneração do disco intervertebral, podendo ocorrer extrusão (Hansen tipo I) ou hérnia (Hansen tipo II) do disco, que por sua vez pode causar compressão da medula ou das raízes nervosas, e até concussão medular (Fingeroth, 1995; Seim III, 2002; Sharp e Wheeler, 2005). Dentre os cães com DDIV, $85 \%$ apresentam a doença na região toracolombar, principalmente entre as vértebras T12-T13-L1. Os discos da região cervical são afetados em aproximadamente $15 \%$ dos casos (Jeffery, 1995; Seim III, 2002).

A apresentação clínica é variável e depende da localização da lesão, do volume de material no interior do canal e da velocidade com que este material é ejetado (Sanders et al., 2002; Amsellem et al., 2003a). Há controvérsias quanto à melhor forma de tratamento da doença. Muitos

Recebido em 12 de julho de 2006 
cães com DDIV podem ser tratados com o uso de analgésicos e antiinflamatórios associados ao repouso absoluto sob confinamento (Seim III, 2002; Davidson, 2003). A DDIV cervical tratada dessa maneira apresenta alta incidência de recidiva ou piora do quadro (Jeffery, 1995; Seim III, 2002; Sharp e Wheeler, 2005). Para cães com DDIV toracolombar com sintomas leves, como dor e ataxia, esse tratamento parece ser o ideal, porém existe o risco de progressão dos sintomas até culminar com paraplegia grave (Jeffery, 1995). Em cães com paraplegia e perda da sensibilidade profunda, o tratamento médico apresenta taxas de sucesso de 7\% (Amsellem et al., 2003b). Mesmo em cães que apresentam quadro leve de DDIV, pode haver grande quantidade de material de disco no interior do canal medular, e para alguns autores, o tratamento ideal da DDIV, em todos os seus graus, seria cirúrgico (Sukhiani et al., 1996; Sharp e Wheeler, 2005).

Os objetivos do tratamento cirúrgico na DDIV são: a descompressão da medula espinhal, a remoção do material do interior do canal medular, a redução do edema, o alívio da dor e a prevenção de futuras extrusões (Tudury, 1985). São comumente descritas duas modalidades de cirurgia para o tratamento da doença de disco no cão: a fenestração e as técnicas descompressivas (Jeffery, 1995; Sharp e Wheeler, 2005). As técnicas descompressivas aplicadas na região toracolombar como a hemilaminectomia, minihemilaminectomia e laminectomia, e na região cervical como a hemilaminectomia e o slot ou fenda ventral, são usadas para remover o material do disco do interior do canal vertebral, principalmente em cães com alterações neurológicas severas, dor e presença de compressão diagnosticada na mielografia (Yovich et al., 1994; Olby et al., 2003; Sharp e Wheller, 2005). Baseando-se nessas informações, o presente trabalho teve como objetivo analisar os sinais clínicos e a resposta ao tratamento cirúrgico descompressivo em 45 cães com DDIV toracolombar ou cervical.

\section{CASUÍSTICA}

Foram estudados 45 casos de DDIV cervical ou toracolombar atendidos entre janeiro de 2001 e dezembro de 2003. Destes, 31 já haviam sido submetidos a tratamento médico sem melhora e foram encaminhados ao Hospital por médicos veterinários para atendimento. Todos os casos foram diagnosticados pela associação dos resultados de exame clínico e neurológico, análise das radiografias simples e contrastadas e exclusão de doenças inflamatórias por meio de análise do líquor. A mielografia foi realizada nos animais anestesiados e com o uso dos contrastes à base de ioversol $^{1}$ ou iohexol ${ }^{2}$, injetados na cisterna magna. Em quatro casos de DDIV toracolombar não foi possível identificar o local correto da compressão, devido ao edema intenso da medula espinhal, realizando-se então uma nova mielografia mediante injeção de contraste na região lombar.

Para que os animais fossem incluídos neste trabalho, foi necessária a confirmação da doença, por meio da visualização do material do disco dentro do canal vertebral durante o ato cirúrgico. Nas afecções cervicais realizou-se o "slot" ou fenda ventral e nas toracolombares realizou-se a hemilaminectomia, técnicas descritas por Sharp e Wheeler (2005). A hemilaminectomia foi associada à fenestração do disco afetado. Os animais com doença de disco cervical foram divididos em três grupos, de acordo com os sinais clínicos apresentados: (A)- hiperpatia; (B)hiperpatia e ataxia; (C)- tetraparesia. Na doença de disco toracolombar o grau de disfunção foi classificado de I a V, segundo Sharp e Wheeler (2005), em que I significa dor toracolombar, sem alterações neurológicas; II, paraparesia ambulatória; III, paraparesia não ambulatória; IV, paraplegia com retenção ou incontinência urinária e $\mathrm{V}$, paraplegia com ausência de dor profunda associada à retenção ou incontinência urinária.

Após a cirurgia, os animais receberam cuidados pós-operatórios intensivos, como analgesia com o uso de opióides, esvaziamento da bexiga, limpeza da pele do abdômen e ao redor do ânus, movimentação passiva dos membros paralisados e auxílio para locomoção utilizando-se uma faixa para suspender o abdômen (Jerram et al., 1997). Os casos foram acompanhados com reavaliações periódicas e após os retornos e alta, por contato telefônico com os proprietários. Para avaliação

\footnotetext{
${ }^{1}$ Optiray, Mallinckrodt Medical AS - Humana Produtos

Hospitalares Ltda - Rio de Janeiro, Brasil

${ }^{2}$ Omnipaque, Nycomed, distribuído por Sanofi Synthelabo -

Rio de Janeiro, Brasil
} 
do resultado final, utilizou-se o seguinte critério: recuperação total - recuperação completa da função motora e urinária e alívio da dor; recuperação parcial - ausência de dor e permanência de alguma disfunção motora ou urinária ; e insucesso - quadro inalterado.

\section{DISCUSSÃO}

Todos os casos apresentaram doença do disco intervertebral do tipo I, ou seja, ruptura do anel fibroso com extrusão do material do disco para o interior do canal vertebral. As raças tiveram distribuição semelhante aos relatos de Brown et al. (1977); Hoerlein (1979), Yovich et al. (1994) e Olby et al. (1994). As ocorrências foram: Dachshund (22/45), seguida pelos cães sem raça definida (7/45), Cocker spaniel (6/45), Lhasa Apso (3/45), Poodle (4/45), Beagle (1/45), Terrier brasileiro $(1 / 45)$ e Pequinês $(1 / 45)$. Os dados referentes ao segmento medular acometido e graus de disfunção encontram-se na Tab. 1.

O diagnóstico da DDIV foi realizado inicialmente com base na história, sinais clínicos e alterações neurológicas. Várias outras doenças, entretanto, devem ser consideradas no rol de diagnósticos diferenciais, como condições inflamatórias, infecciosas, trauma e neoplasias (Hoerlein, 1979; Schulz et al., 1998). A radiografia simples foi utilizada para descartar algumas delas. $\mathrm{Na}$ avaliação das radiografias simples de cães com DDIV podem ser detectados: diminuição do espaço do disco e do forame intervertebral, colapso das articulações sinoviais e disco intervertebral calcificado ou material calcificado dentro do canal medular (Jeffery, 1995). Porém esse exame auxilia na identificação do local exato da lesão somente em 60 a $70 \%$ dos casos (Olby et al., 1994).

A calcificação do núcleo pulposo in situ, decorrente do processo degenerativo do disco, é facilmente detectada nas radiografias simples, e podem existir vários discos calcificados em um mesmo animal, não necessariamente relacionados ao quadro neurológico (Fingeroth, 1995; Jeffery, 1995; Stigen, 1996). Assim, vários autores indicam a realização de mielografia, com ou sem tomografia e ressonância magnética quando disponíveis, para identificação do local correto a ser abordado durante a cirurgia (Jeffery, 1995; Sharp e Wheeler, 2005).

Tabela 1. Grau de lesão em cães com doença do disco intervertebral toracolombar ou cervical e taxa e tempo de recuperação após cirurgia descompressiva

\begin{tabular}{|c|c|c|c|c|c|}
\hline \multicolumn{6}{|c|}{ Resultados } \\
\hline Grau & $\mathrm{N}^{\circ}$ de cães e $\%$ & RT & $\mathrm{TM}$ & $\mathrm{RP}$ & $\mathrm{I}$ \\
\hline \multicolumn{6}{|l|}{ Toracolombar } \\
\hline II & $8(22,2 \%)$ & 6 & 27,3 & 2 & 0 \\
\hline III & $13(36,1 \%)$ & 11 & 16,6 & 2 & 0 \\
\hline IV & $7(19,5 \%)$ & 4 & 15 & 1 & 2 \\
\hline $\mathrm{V}$ & $8(22,2 \%)$ & 5 & 52,8 & 3 & 0 \\
\hline Subtotal & 36 & $26(72,2 \%)$ & & $8(22,2 \%)$ & $2(5,6 \%)$ \\
\hline \multicolumn{6}{|l|}{ Cervical } \\
\hline A & $4(44,5 \%)$ & 4 & 16,8 & 0 & 0 \\
\hline B & $1(11 \%)$ & 1 & 7 & 0 & 0 \\
\hline $\mathrm{C}$ & $4(44,5 \%)$ & 4 & 20 & 0 & 0 \\
\hline Subtotal & 9 & $9(100 \%)$ & & & \\
\hline Total & 45 & $35(77,8 \%)$ & 22,2 & $8(17,8)$ & $2(4,4 \%)$ \\
\hline
\end{tabular}

RT: recuperação total; TM: tempo médio em dias para a recuperação total; RP: recuperação parcial; I: insucesso

A falha da mielografia no diagnóstico da DDIV pode ser decorrente de hemorragia e edema medular resultantes da compressão da medula pelo material do disco, o que dificulta a difusão do meio de contraste (Schulz et al., 1998), fato observado em quatro animais. Nesses casos, observados principalmente na DDIV toracolombar graus IV e V, seguiu-se a recomendação de Olby et al. (1994) e de Jeffery (1995), que preconizam a injeção de contraste 
pela cisterna magna e em seguida pela região lombar, para delimitar a área a ser explorada durante a cirurgia.

É interessante observar que dos oito cães com DDIV toracolombar no grau $\mathrm{V}$, cinco animais $(62,5 \%)$ recuperaram-se totalmente e três $(37,5 \%)$, parcialmente. Destes três, dois permaneceram com discreta ataxia nos membros posteriores e um voltou a caminhar, mas permaneceu com incontinência urinária. Para seus proprietários, porém, o resultado da cirurgia foi considerado bom. A incontinência urinária e/ou fecal pode persistir em cães que recuperaram a função motora, mas nem sempre é percebida como um problema pelos proprietários (Olby et al., 2003). Estes cães foram operados entre dois e cinco dias após o início dos sinais clínicos (média: três dias), sendo seis animais operados mais de 48 horas após o início dos sinais clínicos (média: 3,5 dias) e dois animais, dois dias após o início dos sinais.

Estes resultados estão de acordo com Scott e McKee (1999) e Olby et al. (2003), que observaram taxas de recuperação de 58 a $62 \%$ após realizarem laminectomia ou hemilaminectomia em cães com paraplegia e perda de dor profunda. Segundo esses autores, embora a dor profunda indique lesão medular severa, a perda da função neurológica não é necessariamente irreversível (Scott e McKee, 1999; Olby et al., 2003).

Dos sete animais com DDIV toracolombar no grau IV, quatro $(57,1 \%)$ recuperaram-se totalmente, um (14,3\%) apresentou recuperação parcial, com persistência de leve ataxia nos membros posteriores, e dois $(28,6 \%)$ não apresentaram alteração do quadro inicial. Nos 13 animais com doença de disco toracolombar no grau III, $11(84,6 \%)$ recuperaram-se totalmente e dois $(15,4 \%)$, parcialmente. Assim, de 28 animais com DDIV que apresentavam paraplegia, $20 \quad(71,4 \%)$ recuperaram-se totalmente e seis $(21,4 \%)$ parcialmente.

Em todos os animais submetidos à hemilaminectomia, realizou-se também a fenestração do disco afetado, que parece ser uma prática apropriada, pois permite que resquícios do núcleo pulposo no espaço intervertebral sejam retirados (Fingeroth, 1995). Essa técnica evita o deslocamento do núcleo pulposo remanescente para o interior do canal medular, prevenindo recidiva do quadro. É importante que a descompressão preceda à fenestração, pois essa pode deslocar mais material para o interior do canal, aumentando temporariamente a compressão medular (Fingeroth, 1995; Jeffery, 1995). Knapp et al. (1990), ao estudarem os resultados da fenestração ventral realizada em 160 cães com DDIV toracolombar, constataram que dois cães apresentaram piora do quadro e necessitaram de cirurgia descompressiva para retirar material do disco que sofreu extrusão e comprimiu a medula espinhal, causando lesão irreversível em um animal.

Os animais com DDIV cervical foram tratados com o método da fenda ventral e todos os nove cães $(100 \%)$ recuperaram-se totalmente (Tab. 1) no tempo médio de 14,6 dias. Essa técnica permite recuperação rápida e com poucas complicações. Esse procedimento cirúrgico requer menor dissecção tecidual, traumatiza menos as estruturas anatômicas adjacentes e proporciona a visualização adequada do canal espinhal, com manipulação mínima da medula (Seim III, 2002; Sharp e Wheeler, 2005).

Os cães submetidos à cirurgia descompressiva cervical ou toracolombar apresentaram tempo médio de recuperação total de 22,2 dias. Nos cães com DDIV toracolombar no grau V, o tempo médio para recuperação foi de 52,8 dias, e nos com DDIV cervical do grupo C, 20 dias. Essa diferença na taxa de recuperação é justificada pelo maior espaço existente ao redor da medula espinhal cervical (Jeffery, 1995), e maior espaço para acomodação da medula e do material do disco (Sharp e Wheeler, 2005). Isto também é o motivo de as DDIVs toracolombares nos graus IV e $\mathrm{V}$ serem consideradas emergências cirúrgicas (Schulz et al., 1998) e, portanto, devem ser tratadas precocemente com retirada do material do disco por meio de técnicas descompressivas, para que haja um resultado positivo na recuperação do animal (Amsellem et al., 2003b; Tudury et al., 2004).

Trinta e um cães $(69,0 \%)$ já haviam sido medicados sem sucesso: 11 animais haviam recebido tratamento prévio com antiinflamatórios esteróides, 17 antiinflamatórios não esteróides e três com ambos. Após a realização da cirurgia descompressiva com a retirada do material do interior do canal vertebral, aproximadamente 
$84 \%$ destes animais recuperaram-se totalmente em prazos curtos, resultado similar ao de vários autores (Yovich et al., 1994; Sharp e Wheeler, 2005). O tratamento médico prévio pode ter colaborado, ainda, para a não recuperação nos casos de DDIV no grau IV, devido à demora no encaminhamento dos casos. Nesses casos, pode ainda ter ocorrido concussão medular e alterações irreversíveis no tecido nervoso, e a descompressão medular não alterou o quadro clínico (Jeffery, 1995). Além desses problemas, a demora para realização da cirurgia pode levar ao endurecimento e à adesão do material do disco à dura-máter, dificultando ou impossibilitando sua remoção (Sharp e Wheeler, 2005).

Nos casos de DDIV toracolombar, a diferença entre as médias do tempo de recuperação dos animais que não apresentavam dor profunda e as daqueles que a apresentavam foi de 29,5 dias. A recuperação dos animais com DDIV toracolombar, com ausência de dor profunda (grau V) pode demorar de três a seis meses a mais que a de animais paraplégicos com sensibilidade dolorosa profunda (Amsellem et al., 2003b). Em um estudo feito com 71 cães paraplégicos com dor profunda preservada, o tempo maior de duração dos sinais clínicos até a realização da cirurgia prolongou o tempo de recuperação (Ferreira et al., 2002).

A melhor forma de manejo da DDIV ainda é motivo de discussões. Há relatos de que o tratamento médico seja efetivo em até $71 \%$ dos casos leves, com tempo de recuperação entre três e 12 semanas (Davies e Sharp, 1983). Em cães com DDIV no grau V, apenas $7 \%$ dos casos recuperaram-se com o tratamento médico. Essa diferença sugere que o tratamento cirúrgico deva prevalecer e ser associado ao tratamento conservador principalmente nos casos severos (Amsellem et al., 2003b).

Segundo Jeffery (1995) e Kazakos et al. (2005), o tratamento ideal para a DDIV toracolombar no grau $\mathrm{V}$ permanece controverso. Em muitos casos, devido ao "pré-conceito" existente quanto à não recuperação de cães com DDIV no grau $\mathrm{V}$, os animais não são operados, ou são submetidos à eutanásia (Fingeroth, 1995). Apesar de a avaliação da dor profunda ser subjetiva, apresentar dificuldades de interpretação (Davidson, 2003), e variar de interpretação entre os autores, muitos animais têm potencial para recuperação (Fingeroth, 1995), inclusive quando essa perda tenha mais de 48 horas (Scott e McKee, 1999; Olby et al., 2003; Kazakos et al., 2005) conforme observado em alguns cães deste trabalho. De qualquer modo, cães que apresentam paraplegia e alterações no controle da micção (grau IV) estão próximos de serem grau V, caso haja falha na interpretação, mas em ambos os graus, os animais devem ser operados o mais rápido possível, para evitar danos irreversíveis à medula espinhal (Olby et al., 2003). Existe ainda dificuldade em estimar a duração da perda da sensibilidade profunda, especialmente nos casos referidos (Scott e McKee, 1999; Kazakos et al., 2005).

O prognóstico ainda depende de fatores como a duração dos sinais clínicos, duração do início dos sinais clínicos (Scott e McKee, 1999; Kazakos et al., 2005) e qualidade dos cuidados realizados no período pós-operatório (Jerram et al., 1997), mas a remoção cirúrgica do material extruso parece ter correlação direta com a recuperação, conforme assinalado por McKee (1992). A classificação da DDIV em graus facilitou a escolha do tratamento, permitindo a comparação e a avaliação dos resultados. A cirurgia descompressiva (slot cervical e hemilaminectomia) com a retirada do material do disco do interior do canal vertebral foi uma forma efetiva de tratamento.

\section{REFERÊNCIAS BIBLIOGRÁFICAS:}

AMSELLEM, P.M.; TOOMBS, J.P.; LAVERTY, P.H. et al. Loss of deep pain sensation following thoracolumbar intervertebral disk herniation in dogs: pathophysiology. Comp. Cont. Educ. Pract. Vet., v.25, p.256-264, 2003a.

AMSELLEM, P.M.; TOOMBS, J.P.; LAVERTY, P.H. et al. Loss of deep pain sensation following thoracolumbar intervertebral disk herniation in dogs: treatment and prognosis. Comp. Cont. Educ. Pract. Vet., v.25, p.266-273, 2003b.

BROWN, N.; HELPHREY, M.L.; PRATA, R.G.; Thoracolumbar disk disease in the dog: a retrospective analysis of 187 cases. J. Am. Anim. Hosp. Assoc., v.13, p.665-672, 1977.

DAVIDSON, E.B. Thoracolumbar intervertebral disk disease-to cage or to the OR? Vet. Med., v.98, p.648-649, 2003. 
DAVIES, J.V.; SHARP, J.H. A comparison of conservative treatment and fenestration for thoracolumbar intervertebral disc disease in the dog. J. Small Anim. Pract., v.24, p.721-729, 1983.

FERREIRA， A.J.A.; CORREIA， J.H.D.; JAGGY, A . Thoracolumbar disc disease in 71 paraplegic dogs: influence of rate of onset and duration of clinical signs on treatment results. $J$. Small Anim. Pract., v.43, p.158-163, 2002.

FINGEROTH, J.M. Treatment of canine intervertebral disk disease. Recommendations and controversies. In: BONAGURA, J.D. KIRK's Current veterinary therapy XII. Philadelphia: Saunders, 1995. p.1146-53.

HOERLEIN, B.F. Comparative disk disease: Man and dog. J. Am. Anim. Hosp. Assoc., v.15, p.533-545, 1979.

JEFFERY, N.D. Handbook of small animal spinal surgery. London: Saunders, 1995. 236p.

JERRAM, R.M.; HART, R.C.; SCHULZ, K. Postoperative management of the canine spinal surgery patient - Part I. Comp. Cont. Educ. Pract., Vet. v.19, p.147-161, 1997.

KAZAKOS， G.; POLIZOPOULOU, Z.S.; PATSIKAS, M.N. et al. Duration and severity of clinical signs as prognostic indicators in 30 dogs with thoracolumbar disk disease after surgical decompression. J. Vet. Med., v.52, p.147-152, 2005.

KNAPP, D.P.; POPE, E.R.; HEWETT, J.E. et al. A retrospective study of thoracolumbar disk fenestration in dogs using a ventral approach: 160 cases (1976 to 1986). J. Am. Anim. Hosp. Assoc., v.26, p.543-549, 1990.

McKEE, W.M. A comparasion of hemilaminectomy (with concomitant disc fenestration) and dorsal laminectomy for the treatment of thoracolumbar disc protrusion in dogs. Vet. Rec., v.130, p.296-300, 1992.

OLBY, N.J.; DYCE, J.; HOULTON, J.E.F. Correlation of plain radiographic findings with surgical findings in thoracolumbar disc disease. J. Small Anim. Pract., v.35, p.345-350, 1994.

OLBY, N.; LEVINE, J.; HARRIS, T. et al. Long term functional outcome of dogs with severe injuries of the thoracolumbar spinal cord: 87 cases (1996-2001). J. Am. Vet. Med. Assoc., v.222, p.762-769, 2003.
SANDERS, S.G.; BAGLEY, R.S.; GAVIN, P.R. Intramedullary spinal cord damage associated with intervertebral disk material in a dog. J. Am. Vet. Med. Assoc, v.221, p.1694-1596, 2002.

SCHULZ, K.S.; WALKER, M.; MOON, M. et al. Correlation of clinical, radiographic, and surgical localization of intervertebral disc extrusion in small breed dogs. A prospective study of 50 cases. Vet. Surg., v.27, p.105-111, 1998.

SCOTT, H.W.; McKEE, W.M. Laminectomy for 34 dogs with thoracolumbar intervertebral disc disease and loss of deep pain perception. J. Small Anim. Pract., v.40, p.417-422, 1999.

SEIM III, H.B. Surgery of the cervical spine. In: FOSSUM, T.W. Small animal surgery. Missouri: Mosby, 2002. p.1213-1268.

SHARP, N.J.H.; WHEELER, S.J. Small animal spinal disorders. Edinburgh: Elsevier Mosby, 2005. 379p.

STIGEN, O. Calcification of intervertebral disc in the dachshund: a radiographic study of 115 dogs at 1 and 5 years of age. Acta Vet. Scand., v.37, p.229-237, 1996.

SUKHIANI, H.R.; PARENT, J.M.; ATILOLA, M.A. et al. Intervertebral disk disease in dogs with signs of back pain alone: 25 cases (19861993). J. Am. Vet. Med. Assoc., v.209, p.12751279, 1996.

TUDURY, E.A.; Comparação da abordagem dorsolateral e lateral na região toracolombar, para hemilaminectomia, durotomia e fenestração terapêutica e profilática à protrusão dos discos intervertebrais de cães sadios. 1985. 113f. Dissertação (Mestrado em Medicina Veterinária) Universidade Federal de Santa Maria, Santa Maria, RS.

TUDURY, E.A.; SEVERO, M.S.; MACIEL, M.J. Freqüência de extrusões de núcleos pulposos cervicais e toracolombares, em cadáveres caninos submetidos à técnica de fenestração. Cien. Rural, v.34, p.113-118, 2004.

YOVICH, J.C.; READ, R.; EGER, C. Modified lateral spinal decompression in $61 \mathrm{dogs}$ with thoracolumbar disc protrusion. J. Small Anim. Pract., v.35, p.351-356, 1994. 